\title{
EFECTO DE LA TEMPERATURA DE TERMOTRATAMIENTO EN EL COMPORTAMIENTO ELÉCTRICO DE LA MADERA DE PINO RADIATA
}

\author{
EFFECT OF TEMPERATURE OF THERMOTREATMENT ON \\ ELECTRICAL CONDUCTIVITY OF RADIATA PINE TIMBER
}

\author{
JI. Fernández-Golfín ${ }^{1, \star}$, M. Conde García ${ }^{1}$, JJ. Fernández-Golfín ${ }^{2}$, M. Conde García ${ }^{3}$, \\ E. Hermoso ${ }^{1}$, JC. Cabrero ${ }^{1}$
}

\begin{abstract}
RESUMEN
En el presente trabajo se analiza el efecto de la temperatura del termotratamiento sobre la conductividad eléctrica de la madera de pino radiata. Sobre probetas de madera de pino radiata de procedencia País Vasco (España), termotratada a $190^{\circ} \mathrm{C}$ y $210^{\circ} \mathrm{C}$ por el método Thermowood así como sobre piezas testigo de la misma especie, procedencia y dimensiones, acondicionadas todas ellas hasta masa constante a $20^{\circ} \mathrm{C} / 40 \% \mathrm{HR}, 20^{\circ} \mathrm{C} / 65 \% \mathrm{HR}$ y $20^{\circ} \mathrm{C} / 90 \% \mathrm{HR}$ se evaluó la resistencia eléctrica (longitudinal y transversal) y, posteriormente, se ajustó el modelo Samuelsson para modelizar en cada tipo de material la relación humedad de la madera-resistencia eléctrica. Se concluye que la temperatura empleada en el tratamiento térmico de la madera afecta no sólo a la humedad de equilibrio sino, también, a su conductividad eléctrica, siendo máximo este efecto en el tratamiento efectuado a $210^{\circ} \mathrm{C}$.
\end{abstract}

Palabras clave: Madera termotratada, conductividad eléctrica de la madera, Pinus radiata.

\begin{abstract}
This paper analyzes the effect of heat treatment temperature on the electrical conductivity of radiata pine wood. On specimens of radiata pine of the Basque Country provenance (Spain), heat treated at $190^{\circ} \mathrm{C}$ and $210^{\circ} \mathrm{C}$ by the method Thermowood as well as not treated matched samples, conditioned up to constant mass at the standard conditions of $20^{\circ} \mathrm{C} / 40 \% ; 20^{\circ} \mathrm{C} / 65 \%$ and $20^{\circ} \mathrm{C} / 90 \% \mathrm{RH}$ the electrical resistance (longitudinal and transverse) was measured and a Samuelsson model fitted to describe the relationship between the electrical resistance and moisture content of each material. From the data is concluded that the temperature employed in the thermal treatment timber not only affects equilibrium moisture content of wood but also to its electrical conductivity, being this effect maximum in the processing performed at $210^{\circ} \mathrm{C}$.
\end{abstract}

Keywords: Thermal modified timber, electrical conductivity of timber, Pinus radiata.

\footnotetext{
${ }^{1}$ Centro de Investigación Forestal (CIFOR) del Instituto Nacional de Investigación y Tecnología Agraria y Alimentaria, Madrid (INIA), España.

${ }^{2}$ Escuela Técnica Superior de Ingenieros de Montes, Universidad Politécnica de Madrid. España

${ }^{3}$ Escuela Técnica Superior de Ingenieros Agrónomos y Montes. Universidad de Córdoba. España

^Autor para correspondencia: golfin@inia.es

Recibido: 21.03.2013. Aceptado: 19.04. 2013.
} 


\section{INTRODUCCIÓN}

La norma experimental UNE-CEN/TS 15679EX:2009 titulada "Madera modificada térmicamente (MMT): definiciones y características", define la madera termotratatada (MMT) como "Madera en la cual la composición de las paredes celulares y las propiedades físicas se modifican por exposición a una temperatura mayor de $160^{\circ} \mathrm{C}$ y a condiciones de disponibilidad reducida de oxígeno. La madera se modifica de tal forma que como mínimo algunas propiedades quedan alteradas de forma permanente y en toda la sección de la pieza”.

La influencia de la temperatura de tratamiento en la higroscopicidad y estabilidad dimensional de la madera ha sido constatada desde los primeros estudios sobre secado industrial de la madera (Tiemann 1915, Koehler y Pillow 1925, Greenhill 1936).

Los primeros trabajos específicamente orientados hacia el termotratamiento son los debidos a Stamm y Hansen (1937) quienes trabajando sobre pino blanco americano (Pinus strobus), estudiaron el efecto de la temperatura de calentamiento en presencia de diversas atmósferas gaseosas, concluyendo que el aumento de la temperatura y del tiempo de calentamiento incrementaban la estabilidad de la madera.

De interés para la completa comprensión de los orígenes de la técnica de modificación de la madera es el extenso trabajo de Seborg et al. (1962) quienes analizaron el estado del arte de las técnicas químicas y físicas existentes hasta el momento para la estabilización de la madera, muchas de las cuales se basaban en la introducción y posterior polimerización o fijación de resinas en el interior de la madera (Compreg, Impreg, Staypack, etc.). De más reciente realización pero con idéntico interés bibliográfico para entender el estado del arte son los trabajos de Rapp (2001) y Hill (2006).

Los más recientes avances tecnológicos sobre la modificación térmica de la madera han llevado al desarrollo de las técnicas conocidas como Thermowood (Viitaniemi et al. 1997), Torrefacción o retificación (Weiland y Guyonnet 1997) y Plato process (Tjeerdsma et al. 1998). Estas tres técnicas pueden ser encuadradas dentro del grupo de modificación hidrotérmica (efecto combinado de temperatura y humedad), siendo muy diferentes en su ejecución y resultados de aquellas otras que como el método OHT (Sailer et al. 2000) plantean un calentamiento de la madera en el seno de un autoclave completamente relleno de aceites vegetales de diversa naturaleza (lino, colza, etc.).

Centrándonos en los métodos hidrotérmicos anteriormente mencionados, son los métodos denominados como Thermowod y PLATO los más ampliamente utilizados por la industria y los que, por tanto, mayores volúmenes de madera suministran al mercado. Ambos métodos son especialmente adecuados para el tratamiento de maderas de alta (pino silvestre) o muy alta permeabilidad (pino radiata) aunque las condiciones más suaves del tratamiento PLATO hacen que las colaterales pérdidas de propiedades mecánicas sean más reducidas, lo que en determinados usos puede ser una ventaja. Por el contrario, el método Thermowood tiene un notable menor coste de inversión y una más sencilla operación.

Trabajando con el método Thermowood sobre picea y diversas especies de alerce, Frühwald (2007) concluyó que la velocidad de la sorción y la humedad de equilibrio higroscópico decrecían con la temperatura empleada para el tratamiento térmico $\left(190^{\circ} \mathrm{C}\right.$ y $\left.210^{\circ} \mathrm{C}\right)$. Esta misma autora comprobó que la cuantía de la reducción era distinta según la especie, poniendo claramente de manifiesto la influencia de esta variable en la modificación de las relaciones agua-madera. 
La influencia que en el resultado final tienen variables tales como la especie y la metodología del tratamiento queda claramente reflejada en el articulado de la norma experimental UNE-CEN/TS 15679EX ya que en su apartado 1 se establece textualmente "Las propiedades de la madera modificada térmicamente y el alcance de la modificación de sus propiedades, dependen de la especie de madera, del tipo de tecnología y de los parámetros del tratamiento, en particular de la temperatura del tratamiento". Todo ello indica la necesidad de que con carácter previo a su puesta en mercado, toda la madera termotratada de cada especie y por cada método sea sometida a un completo proceso de caracterización que contemple un amplio abanico de propiedades físicas y mecánicas (parcialmente contemplado en la citada norma UNE-CEN/TS 15679EX que en este sentido sirve como primera guía para llevar a cabo dicho proceso de caracterización) y el efecto que en ellas tengan las posibles variables de proceso.

Diversos estudios llevados al efecto y analizados en profundidad por Rowell et al (2002) han puesto de manifiesto que el tratamiento de la madera con altas temperaturas produce cambios sustanciales en sus propiedades químicas, afectando especialmente a las hemicelulosas, a la lignina y a la estructura cristalina interna de las cadenas de celulosas. Es evidente que si la estructura y composición química internas de la madera se ven alteradas por los tratamientos con calor también puede serlo su conductividad eléctrica, lo que habría de expresarse en diferentes relaciones entre el contenido de humedad y la resistencia eléctrica del material, relaciones que son la base del método industrial de estimación de la humedad de la madera mediante medidas de resistencia eléctrica (xilohigrómetros de resistencia).

El empleo de medidores portátiles de la humedad de la madera mediante métodos eléctricos (xilohigrómetros) está ampliamente generalizado en la industria de la madera así como en todas aquellas aplicaciones en las que la precisión no sea una exigencia acuciante (la precisión de un aparato basado en la medida de la resistencia eléctrica correctamente calibrado ronda el $1 \%$ en valor absoluto, mientras que la de uno que funcione por el método de capacitancia difícilmente bajará del 2-3\%). Dentro de los equipos portátiles de humedad de la madera son los que se basan en medidas de la resistencia eléctrica los que más habitualmente son usados en el control de calidad de productos en parque, almacén y obra.

Una de las más importantes fuentes de error cuando se estima la humedad de la madera mediante el empleo de equipos de medida de la resistencia eléctrica, es el uso de equipos que no incorporan los sistemas adecuados para la corrección interna de las lecturas por el efecto de la especie o de las características del sustrato (por ejemplo, madera tratada químicamente, madera termotratada, etc.). Es muy corriente que la transformación de las medidas de resistencia eléctrica (las que efectúa el aparato) en medidas de humedad de madera (las lecturas que aporta el aparato) se efectúen internamente en el aparato mediante el empleo de una curva interna de conversión obtenida mediante el ensayo en laboratorio sobre una única especie de madera y posterior "corrección" de dichas medidas mediante diversos sistemas para ajustar el efecto de la especie. Detalle de algunos factores de corrección por el efecto de la especie pueden verse en los trabajos de Blakemore (2003), Gillis et al. (2001), Forsén y Tarvainen (2000) y Langwig y Skaar (1975).

Sobre la existencia de imprecisiones debidas al mal ajuste interno de los equipos, Vermaas (1982) reportó que equipos de medida de la humedad de la madera de origen europeo que incorporaban una curva interna de conversión basada en la madera de picea o de pino europeo, generaban notables imprecisiones cuando eran empleados para evaluar la humedad de las maderas de pino radiata o pino pinaster de origen sudafricano.

Por otra parte, el vigente Reglamento Comunitario de Productos de Construcción que impone el Marcado CE en muchas familias de productos de la madera (madera aserrada estructural, madera laminada encolada, ventanas, suelos, etc.) de acuerdo con los métodos contemplados en cada una de sus normas armonizadas, exige que cada proceso industrial posea su sistema interno de Control de la Producción en Fábrica en el que la medida de la humedad de la madera suele jugar un papel destacado. 
Así, por ejemplo, la norma armonizada UNE-EN 14081-1:2006+A1:2011 sobre madera aserrada de sección rectangular destinada al uso estructural, exige de forma taxativa que los equipos de medida no destructiva de la humedad sean calibrados anualmente. La calibración de tales equipos de medida (cuando son de la tecnología de resistencia eléctrica) sólo es posible llevarla a cabo especie a especie y para ejecutar esta labor de forma rápida han de emplearse cajas de décadas junto con las curvas que relacionan entre sí la resistencia eléctrica con la humedad de la madera.

El objetivo del presente trabajo consiste, en primera instancia, en analizar la influencia que el termotratamiento tiene en la resistencia eléctrica de la madera de pino radiata y, en segunda instancia, en proponer las curvas más adecuadas para la conversión de las medidas de resistencia eléctrica en medidas de humedad de madera para la madera termotratada de pino radiata mediante inyección de vapor (sistema Thermowood) a $190^{\circ} \mathrm{C}$ y $210^{\circ} \mathrm{C}$.

Entre los modelos que se han usado para expresar matemáticamente la relación entre la humedad de la madera y su resistencia eléctrica es el modelo de Samuelsson (1990) el que más ampliamente ha sido empleado en los últimos trabajos (Forsén y Tarvainen 2000, Fernández-Golfín et al. 2012) ya que permite unos ajustes muy adecuados para su posterior uso predictivo. Este modelo se expresa como sigue:

$$
\log [\log (\mathrm{R})+1]=\mathrm{a}+\mathrm{b} \cdot \mathrm{h}
$$

donde:

$\mathrm{R}$ es la Resistencia eléctrica de la madera (en Mohms)

a y b son los coeficientes de la regresión

$\mathrm{h}$ es el contenido de humedad de la madera (en \%).

La obtención de tales curvas será de utilidad a la industria para la correcta calibración de los equipos de estima de la humedad de la madera por métodos eléctricos que hayan de ser usados sobre madera termotratada de pino radiata así como para el diseño de equipos específicamente destinados a trabajar íntegramente sobre madera de pino radiata, termotratada o no.

\section{METODOLOGÍA}

\section{Material de ensayo}

El trabajo se llevó a cabo sobre madera aserrada de pino radiata de procedencia País Vasco (NE de España). Para ello se seleccionaron aleatoriamente tablones de corte tangencial de $50 \mathrm{~mm} \times 200 \mathrm{~mm}$ de sección, sin tratar y termotratados a $190^{\circ} \mathrm{C}$ y a $210^{\circ} \mathrm{C}$ mediante un proceso industrial llevado a cabo por la empresa Termogenik en sus instalaciones de Orozco (Vizcaya), de acuerdo con la metodología y equipamiento correspondiente al sistema conocido como Thermowood.

Los tablones de madera se cortaron en muestras de ensayo de aproximadamente $50 \mathrm{~mm}$ de largo, $50 \mathrm{~mm}$ de ancho y $20 \mathrm{~mm}$ de espesor. Por lo menos $50 \mathrm{~cm}$ fueron descartados de cada extremo de los tablones antes de cortar las muestras de ensayo. Se seleccionaron al azar 36 muestras de ensayo para cada tipo de material, denominando a cada grupo como PRST (pino radiata sin tratar), PRTWS (pino radiata termotratado a $190^{\circ} \mathrm{C}$ ) y PRTWD (pino radiata termotratado a $210^{\circ} \mathrm{C}$ ). Todas las probetas de ensayo fueron elegidas libres de defectos visibles, tales como nudos, bolsas de resina, elevadas inclinaciones de fibras, manchas, etc.

Tras su obtención y selección, nueve probetas de ensayo de cada grupo fueron introducidas en tres cámaras climáticas distintas en las que reinaban una misma temperatura de $20 \pm 0,5^{\circ} \mathrm{C}$ y humedades relativas de $40 \pm 5 \%, 65 \pm 5 \%$ y $90 \pm 5 \%$, respectivamente. Probetas del material sin tratar también fueron introducidas en una cuarta condición de humedad relativa de $80 \pm 5 \%$. 
La masa de cada una de las probetas fue controlada semanalmente, registrando su ganancia o pérdida semanal. Para evitar el efecto de los gradientes internos de humedad en las mediciones de resistencia eléctrica, las probetas de ensayo se mantuvieron en las cámaras hasta alcanzar masa constante, lo cual se consideró que ocurría cuando la variación de masa semanal fue $\leq 0,1 \%$ (como media después de cuatro meses en la cámara). Todas las masas se determinaron utilizando una balanza METTLER TOLEDO (Delta Range PB 303) con una resolución de 0,001 g.

Tanto las cámaras de acondicionamiento como la balanza empleadas fueron sometidas a calibración y mantenimiento periódico de acuerdo con los procedimientos internos incorporados en el Manual de la Calidad adoptado por nuestro laboratorio para el trabajo (acreditado por ENAC) según ISO 17025.

\section{Caracterización de la variación de la resistencia eléctrica con la humedad de la madera}

Al final del periodo de acondicionamiento, la resistencia eléctrica de cada probeta fue medida haciendo uso de un medidor de alta resistencia AGILENT 4339B (rango $10^{3}-10^{15} \Omega$, precisión de $0,5 \%$, resolución de visualización 5 dígitos). Las especificaciones de medición fueron las siguientes:

- $\quad$ Voltaje de medida: $10 \mathrm{~V}$

- $\quad$ Temperatura del material en el momento de la medida (material y de laboratorio): $20 \pm 0,5^{\circ} \mathrm{C}$

- $\quad$ Retardo de tiempo de medición: $5 \mathrm{~s}$

Estas especificaciones de medida fueron adoptadas por ser las mismas que las utilizados por Forsén y Tarvainen (2000) y Fernández-Golfín et al. (2012), de forma y manera que los resultados en el presente trabajo fueran compatibles con los obtenidos de aquellos.

Las medidas de resistencia eléctrica se efectuaron tanto longitudinal (Rp) como transversalmente (Rt) a la fibra de la madera, introduciendo para ello hasta el centro de cada probeta dos electrodos en dirección radial y otros dos en dirección transversal. Los electrodos consistieron en clavos de acero inoxidable de $10 \mathrm{~mm}$ de longitud y 2,6 $\mathrm{mm}$ de diámetro, separados entre sí $28,5 \mathrm{~mm}$. Los electrodos fueron introducidos por la cara tangencial (es decir, perpendicularmente con respecto a los anillos) hasta el centro de las piezas (Figura 1). La resistencia eléctrica de la madera, por tanto, se midió tanto paralelamente a la fibra (Rp) (como se recomienda en la norma UNE-EN 13183-2:2002) como perpendicularmente (transversalmente) a la fibra (Rt).

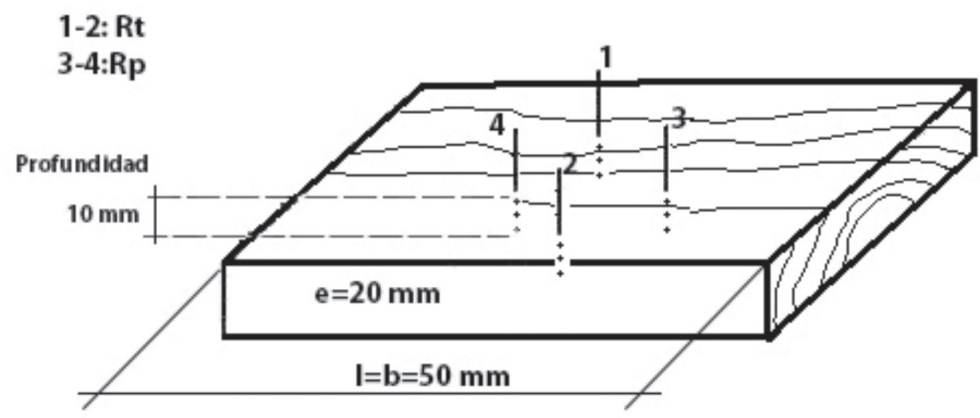

Figura 1. Disposición de medida. 
Todas las mediciones de resistencia fueron efectuadas tras la verificación del buen funcionamiento del medidor de alta resistencia, haciendo uso de una caja de décadas TINSLEY 4721 (en posesión de un certificado de calibración externa según ISO 17025).

Para evitar el efecto que en la medida de la resistencia pudieran tener los cambios superficiales del contenido de humedad o de la temperatura, todas las lecturas eléctricas fueron tomadas sobre las probetas situadas en el interior de sus cámaras de acondicionamiento.

Tras efectuar las medidas de resistencia eléctrica las probetas fueron pesadas (peso húmedo [wh]) con una demora de no más de 1 min y, posteriormente, introducidas en una estufa para su secado hasta masa constante a una temperatura de $103 \pm 2^{\circ} \mathrm{C}$, momento en el que para cada probeta se registró su masa anhidra (wd). El contenido de humedad (h) de cada probeta fue calculado (en porcentaje del peso en seco) de acuerdo con la siguiente expresión:

donde:

$$
\mathrm{h}=\left[\left(\mathrm{w}_{\mathrm{h}}-\mathrm{w}_{\mathrm{d})} / \mathrm{w}_{\mathrm{d}}\right] \cdot 100\right.
$$

$\mathrm{W}_{\mathrm{h}}$ es la masa húmeda $(\mathrm{g})$.

$\mathrm{w}_{\mathrm{d}}$ es la masa seca $(\mathrm{g})$.

\section{Ajuste de modelos}

El ajuste del modelo de Samuelsson (ecuación 1) a los pares de valores humedad-resistencia eléctrica se llevo a cabo mediante regresión lineal utilizando el software estadístico Statgraphics Centurion XV.

\section{RESULTADOS Y DISCUSIÓN}

La tabla 1 muestra los coeficientes de regresión (a y b) del modelo Samuelsson, utilizando Log [Log $(\mathrm{R})+1$ ] como variable dependiente y el contenido de humedad (h) como variable independiente. Para todas las rectas de regresión, la tabla 1 también aporta el valor del coeficiente de determinación $\mathrm{R}^{2}$, el cual en todos los casos fue muy alto $(>0,95)$.

Tabla 1. Coeficientes de regresión para el modelo de Samuelsson.

\begin{tabular}{|c|c|c|c|c|c|c|c|}
\hline \multirow{2}{*}{ Material } & \multirow{2}{*}{$\begin{array}{c}\mathbf{N}^{\circ} \\
\text { probetas }\end{array}$} & \multicolumn{3}{|c|}{ Paralela a la fibra (Rp) } & \multicolumn{3}{|c|}{ Perpendicular a la fibra (Rt) } \\
\hline & & a & b & $\overline{\mathbf{R}^{2}}$ & a & b & $\overline{\mathbf{R}^{2}}$ \\
\hline Sin tratar (PRST) & 36 & $1,11945 \pm 0,00667559$ & $-0,0414002 \pm 0,00046$ & 99,4 & $1,12721 \pm 0,00792254$ & $-0,0409546 \pm 0,00055$ & 99,2 \\
\hline PRTWS $\left(190^{\circ} \mathrm{C}\right)$ & 36 & $1,0346 \pm 0,00708421$ & $-0,0388926 \pm 0,00058$ & 99,4 & $1,03532 \pm 0,00594226$ & $-0,0376629 \pm 0,00049$ & 99,6 \\
\hline PRTWD $\left(210^{\circ} \mathrm{C}\right)$ & 36 & $1,08884 \pm 0,00419287$ & $-0,0462147 \pm 0,00044$ & 99,7 & $1,07715 \pm 0,00499295$ & $-0,0442112 \pm 0,00053$ & 99,6 \\
\hline
\end{tabular}

La tabla 2 recoge los valores de humedad de equilibrio y de resistencia eléctrica medidos paralela y transversalmente para cada tipo de material en cada condición de ensayo. 
Tabla 2. Humedad de equilibrio y resistencia eléctrica por dirección, condición y tipo de material.

\begin{tabular}{|c|c|c|c|c|c|c|c|c|c|}
\hline \multirow{2}{*}{ Material } & \multicolumn{3}{|c|}{$20^{\circ} \mathrm{C} / 40 \% \mathrm{HR}$} & \multicolumn{3}{|c|}{$20^{\circ} \mathrm{C} / 65 \% \mathrm{HR}$} & \multicolumn{3}{|c|}{$20^{\circ} \mathrm{C} / 90 \% \mathrm{HR}$} \\
\hline & $\begin{array}{c}\text { h } \\
(\%)\end{array}$ & $\begin{array}{c}\mathbf{R p} \\
(\mathbf{M o h m})\end{array}$ & $\begin{array}{c}\text { Rt } \\
(\text { Mohm) }\end{array}$ & $\begin{array}{c}\text { h } \\
(\%)\end{array}$ & $\begin{array}{c}\text { Rp } \\
(\mathbf{M o h m})\end{array}$ & $\begin{array}{c}\mathbf{R t} \\
(\mathbf{M o h m})\end{array}$ & $\begin{array}{c}\text { h } \\
(\%)\end{array}$ & $\begin{array}{c}\mathbf{R p} \\
(\mathbf{M o h m})\end{array}$ & $\begin{array}{c}\text { Rt } \\
(\text { Mohm) }\end{array}$ \\
\hline Sin tratar (PRST) & 9,2 & 31866 & 55383 & 12,6 & 704 & 833 & 20,2 & 11,1 & 13,1 \\
\hline $\operatorname{PRTWS}\left(190^{\circ}\right)$ & 7,5 & 45624 & 61121 & 9,5 & 3697 & 4941 & 17,8 & 15,3 & 20,1 \\
\hline $\operatorname{PRTWD}\left(210^{\circ}\right)$ & 6,4 & 295466 & 371740 & 7,7 & 28617 & 36520 & 13,4 & 91,7 & 115,7 \\
\hline
\end{tabular}

Del análisis de los datos de la tabla 2 lo primero que parece desprenderse es que, como era de esperar, el termotratamiento de la madera de pino radiata modifica su comportamiento higroscópico y eléctrico, especialmente si dicho tratamiento se efectúa a $210^{\circ} \mathrm{C}$. Así es especialmente patente el notable incremento que, a igualdad de condiciones higrotérmicas en el medio circundante, en la resistencia eléctrica introduce el termotratamiento a $210^{\circ} \mathrm{C}$.

Este hecho queda reflejado gráficamente en las figuras 2 a 4, que recogen los gráficos de "cajas y bigotes" correspondientes al test ANOVA implementado sobre las medidas de resistencia eléctrica longitudinal de cada tipo de material y en cada condición higrotérmica (el análisis sobre las medidas transversales produce idénticos resultados, se omite por brevedad expositiva).

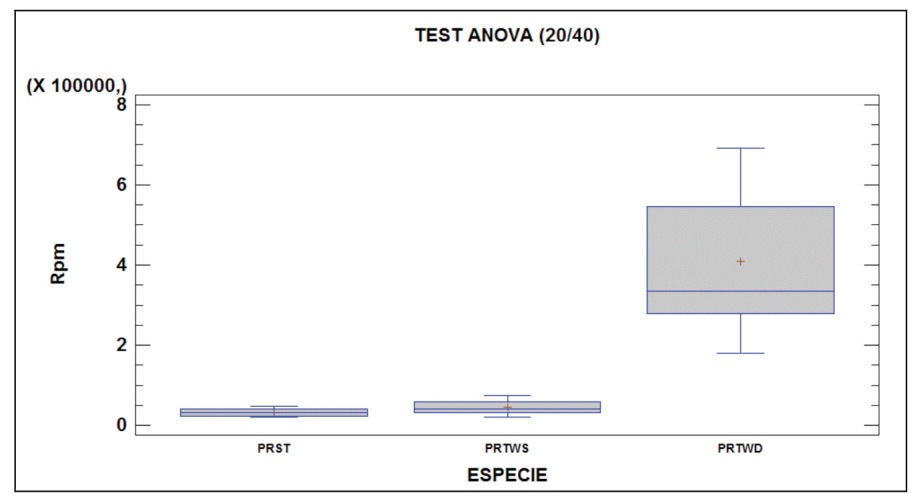

Figura 2. Test ANOVA para las medidas longitudinales efectuadas a $20 / 40\left({ }^{\circ} \mathrm{C} / \%\right)$.

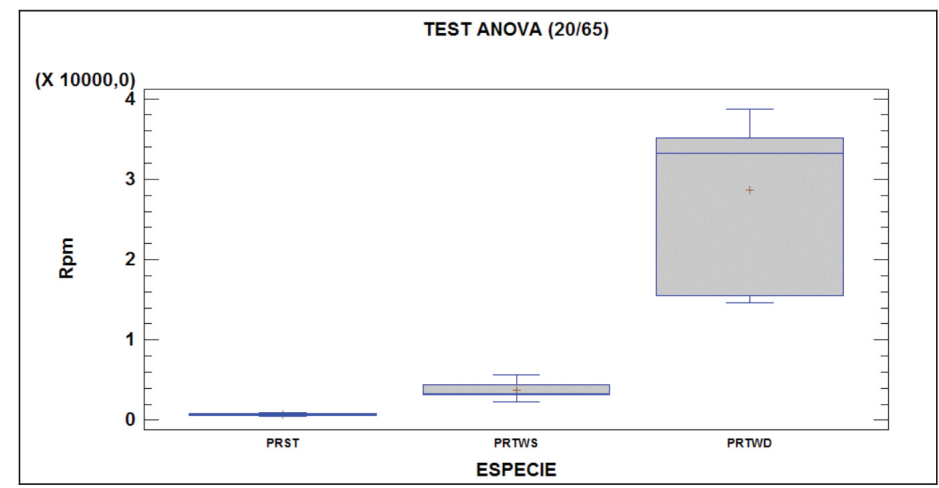

Figura 3. Test ANOVA para las medidas longitudinales efectuadas a $20 / 65\left({ }^{\circ} \mathrm{C} / \%\right)$. 


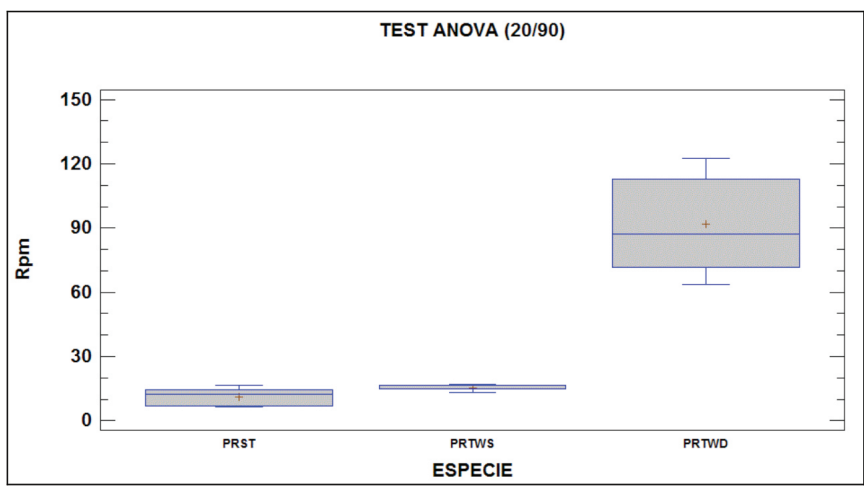

Figura 4. Test ANOVA para las medidas longitudinales efectuadas a $20 / 90\left({ }^{\circ} \mathrm{C} / \%\right)$.

Del análisis de las figuras 2 a 4 se desprende que sólo el material tratado a $210^{\circ} \mathrm{C}$ (PRTWD) exhibe un comportamiento estadísticamente distinto en todas las condiciones higrotérmicas al de la madera sin tatar y tratada a $190^{\circ} \mathrm{C}$ (PRTWS).

En la tabla 3 quedan recogidos los valores reales y estimados de la humedad para cada tipo de material y condición empleando los modelos de la tabla 1.

Tabla 3. Valores estimados y reales de la humedad para cada condición y tipo de madera.

\begin{tabular}{|l|c|c|c|c|c|c|}
\hline \multirow{2}{*}{ Condición } & \multicolumn{2}{|c|}{ Sin tratar (PRST) } & \multicolumn{2}{c|}{ PRTWS } & \multicolumn{2}{c|}{ PRTWD } \\
\cline { 2 - 7 } & Estimada & Real & Estimada & Real & Estimada & Real \\
\hline & \multicolumn{5}{|c|}{ MEDIDAS LONGITUDINALES } \\
\hline $\mathbf{2 0 / 4 0}$ & 9,2 & 9,2 & 7,2 & 7,5 & 6,0 & 6,4 \\
\hline $\mathbf{2 0 / 6 5}$ & 12,9 & 12,6 & 9,6 & 9,5 & 7,6 & 7,7 \\
\hline $\mathbf{2 0 / 9 0}$ & 19,5 & 20,2 & 17,9 & 17,8 & 13,4 & 13,4 \\
\hline & 9,0 & 9,2 & 7,2 & 7,5 & 5,9 & 6,4 \\
\hline $\mathbf{2 0 / 4 0}$ & 13,0 & 12,6 & 9,7 & 9,5 & 7,5 & 7,7 \\
\hline $\mathbf{2 0} / \mathbf{6 5}$ & 19,6 & 20,2 & 17,9 & 17,8 & 13,4 & 13,4 \\
\hline $\mathbf{2 0} / \mathbf{9 0}$ & \multicolumn{7}{|c|}{ MEDIDAS TRANSVERSALES } \\
\hline
\end{tabular}

El análisis de los datos de la tabla 3 nos permite observar que los modelos de la tabla 1 predicen muy bien (errores inferiores al $0,7 \%$ ) los valores medios reales de la humedad de cada material en cada condición y forma de medida (longitudinal y transversal).

En la tabla 4 quedan recogidos los valores estimados para la resistencia paralela ( $R p)$ de los tres tipos de materiales en función de la humedad de la madera 
Tabla 4. Variación de los valores estimados de la resistencia eléctrica (long.) con la humedad para los distintos tipos de material.

\begin{tabular}{|c|c|c|c|}
\hline \multirow{2}{*}{$\begin{array}{c}\text { Humedad } \\
\%\end{array}$} & \multicolumn{3}{|c|}{ Resistencia paralela (Mohm) } \\
\cline { 2 - 4 } & Sin tratar & PRWTS & PRWTD \\
\hline 8 & 138399 & 19500 & 17277 \\
\hline 9 & 38260 & 6869 & 5114 \\
\hline 10 & 11889 & 2646 & 1712 \\
\hline 11 & 4109 & 1106 & 640 \\
\hline 12 & 1564 & 498 & 264 \\
\hline 13 & 650 & 240 & 119 \\
\hline 14 & 293 & 123 & 58 \\
\hline 15 & 142 & 67 & 31 \\
\hline 16 & 73 & 38 & 17 \\
\hline 17 & 40 & 23 & 10 \\
\hline 18 & 23 & 14 & 6 \\
\hline 19 & 14 & 9 & 4 \\
\hline 20 & 9 & 6 & 3 \\
\hline
\end{tabular}

Tal y como se deriva del análisis de los datos de la tabla 4 anterior, se observa un aumento de la conductividad eléctrica con el tratamiento térmico a valores dados de humedad de madera.

Este último comportamiento debería ser objeto de investigaciones posteriores ya que podría estar en la línea de algunos de los trabajos referenciados por Rowell et al. (2002), según los cuales el principal efecto de la alta temperatura sobre la estructura y propiedades químicas de la madera sería la modificación del grado de cristalinidad de las celulosas, lo que haría que los caminos para la conducción eléctrica en el seno de la madera fueran los mismos pero dotados de una mayor conductividad eléctrica, lo que se manifestaría en resistencias eléctricas de menor cuantía a igualdad de humedad del material, tal y como se aprecia en la tabla 4. 


\section{CONCLUSIONES}

Los resultados obtenidos demuestran que la temperatura a la que se efectúa el termotratamiento afecta no sólo a la higroscopicidad de la madera sino, también, a la conductividad eléctrica. Estos mismos resultados vienen a confirmar lo ya visto en otros trabajos sobre el efecto de las altas temperaturas de tratamiento en las propiedades mecánicas del material (Frühwald 2007) en el sentido de que parece existir una notable diferencia entre el efecto de $190^{\circ} \mathrm{C}$ y el de $210^{\circ} \mathrm{C}$.

El mayor efecto de la temperatura de tratamiento de $210^{\circ} \mathrm{C}$ sobre la higroscopicidad y relaciones agua-madera del material sólo podrá ser considerado a la luz del efecto colateral que se producen en el resto de propiedades físico-mecánicas y la durabilidad, aspecto éste que será abordado en un trabajo posterior de este mismo grupo de investigación.

\section{AGRADECIMIENTOS}

El presente trabajo fue parcialmente financiado por el Plan Nacional de I+D+i español y ejecutado en el seno del proyecto RTA 2008-00005. Los autores desean, así mismo, expresar su gratitud a la empresa Termogenik que cedió el material de ensayo. 


\section{BIBLIOGRAFÍA}

Blakemore, P. 2003. The use of hand-held electrical moisture meters with commercially important Australian hardwoods- Part 1 - Executive summary, methods, results, conclusion \& recommendations. Forest \& Wood Products Research \& Development Corporation. Australian Government. Project No. PN01.1306. 88p.

Comité Europeo de Normalización, UNE-CEN. 2009 Madera modificada térmicamente (MMT): definiciones y características. UNE-CEN/TS 15679EX:2009. AENOR.

Comité Europeo de Normalización, UNE-CEN. 2002. Moisture content of a piece of sawn timber. Part 2: Estimation by electrical resistance method. UNE-EN 13183-2:2002. AENOR.

Comité Europeo de Normalización, UNE-EN. 2011. Estructuras de madera. Madera estructural con sección transversal rectangular clasificada por su resistencia. Parte 1: Requisitos generales. UNEEN 14081-1:2006A1:2011. AENOR.

Fernández-Golfín, J.I.; Conde García, M.; Conde García, M.; Fernández-Golfín, J.J.; Calvo Haro, R.; Baonza Merino, M.V.; De Palacios De Palacios, P. 2012. Curves for the estimation of the wood moisture content of ten hardwoods by means of electrical resistance measurements. Forest Systems 21(1): 121-127.

Forsén, H.; Tarvainen, V. 2000. Accuracy and functionality of hand held wood moisture content meters. VTT publications num 420. 95 pp. ISBN 951-38-5581-3.

Frühwald, E. 2007. Effect of high-temperature drying on properties of Norway spruce and larch. Holz als Roh-und Werkst 65: 411-418.

Gillis, C.M.; Stephens, W.C.; Peralta, P.N. 2001. Moisture meter correction factors for four Brazilian wood species. For Prod J 5(4): 83-86.

Greenhill, W.L. 1936. Damping capacity of timber. J Council Sci Ind Research 9: 265-276

Hill, C.A.S. 2006. Wood modification: chemical, thermal and other processes. Wiley series in renewable resources. J. Wiley and Sons. ISBN-10: 0-470-02172-1.

Koehler, A.; Pillow, M.Y. 1925. Effect of high temperatures on the mode of fracture of a softwood. Southern Lumberman 121(1576): 219-221.

Langwig, J.E.; Skaar, C. 1975. Electrical resistivities of 18 Venezuelan and 13 North American woods. Wood Sci 8(1): 350-354.

Rapp, A.O. 2001. Review of heat treatments of wood. Proceedings of the special seminar COST E-22 held in Antibes, France. 9 February 2001. European Commission, 64. ISBN 3-926301-02-3.

Rowell, R.; Lange, S.; Mesweeny, J.; Davis, M. 2002. Modification of wood fiber using steam. In Proceedings of The $6^{\text {th }}$ Pacific Rim Bio-based composites symposium. Workshop on the Chemical modification of cellulosics. Portland, Oregon, USA. pp: 606-615.

Sailer, M.; Rapp, A.O.; Leithoff, H.; Peek, R.D. 2000. Vergütung von Holz durch Anwendung einer Öl-Hitzebehandlung. Holz als Roh-und Werkst 58: 15-22. 
Samuelsson, A. 1990. Resistanskurvor für elektriska fuktkvotsmätare. Träteknik Centrum, Rapport L 9006029. Stockholm. 37 pp.

Seborg, R.M.; Tarkow, H.; Stamm, A.J. 1962. Modified woods. Forest Prod. Lab. Report $n^{\circ} 2192$ (version revisada). $31 \mathrm{pp}$.

Stamm, A.J.; Hansen, L.A. 1937. Minimizing Wood Shrinkage and Swelling: Effect of Heating in Various Gases. Ind Eng Chem 29 (7): 831-833.

Tiemann, H.D. 1915. The effect of different methods of Drying on the Strength of wood. Lumber World Review 28(7): 19-20.

Tjeerdsma, B.F.; Boonstra, M., Militz, H. 1998. Thermal modification of non-durable wood species. 2. Improved wood properties of thermal treated wood. IRG document IRG/WP 98-40123.

Vermaas, H.F. 1982. D.C. Resistance moisture meters for wood. Part I.: review of some fundamentals considerations. South African Forestry Journal 121: 88-92.

Viitaniemi, P.; Jämsä, S.; Viitanen, H. 1997. Method for improving biodegradation resistance and dimensional stability of cellulosic products. USA patent $\mathrm{n}^{\circ} 5678324$. Official Gazette of the United States Patent and Trademark Office: Patents, Volumen 1203, Número 3.

Weiland, J.J.; Guyonnet, R. 1997. Retifiziertes Holz (retification process for wood). In: Verdichterter Holzbau in Europa. Motivation, Erfahrung, Entwicklung. Dreilander Holztagung. Joanneum Research Fachtage. 2-5 November. Grazer Congress, Graz, Austria. 